
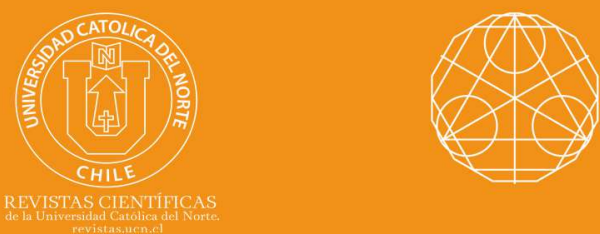

d oi" 10.22199/issn.0717-6279-4073

\title{
A subclass with bi-univalence involving Horadam Polynomials and its coefficient bounds
}

\section{K. Muthunagai ${ }^{1}$ (D) orcid.org/0000-0002-3020-7649 \\ G. Saravanan ${ }^{2}$ (D orcid.org/0000-0002-5706-4174 \\ S. Baskaran ${ }^{3}$ @ orcid.org/0000-0001-8980-9671}

${ }^{1}$ Vellore Institute of Technology, School of Advanced Sciences, Chennai, TN, India.

muthunagai@vit.ac.in

${ }^{2}$ Patrician College of Arts and Science, Dept. of Mathematics, Chennai, TN, India.

varan825@yahoo.com

${ }^{3}$ Agurchand Manmull Jain College, Dept. of Mathematics, Chennai, TN, India.

•sbas9991@gmail.com

Received: 02 April 2020 | Accepted: 19 December 2020

\section{Abstract:}

In this research contribution, we have constructed a subclass of analytic bi-univalent functions using Horadam polynomials. Bounds for certain coefficients and FeketeSzegö inequalities have been estimated.

Keywords: Analytic functions; Bi-univalent functions; Horadam polynomials.

MSC (2020): 30C45, 30C15.

\section{Cite this article as (IEEE citation style):}

K. Muthunagai, G. Saravanan, and S. Baskaran, "A subclass with bi-univalence involving Horadam Polynomials and its coefficient bounds", Proyecciones (Antofagasta, On line), vol. 40, no. 3, pp. 721-730, 2021, doi: 10.22199/issn.0717-6279-4073

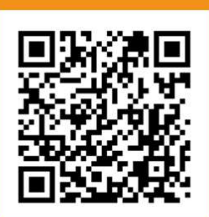

Article copyright: (C) 2021 Krishnan Muthunagai, G. Saravanan, and S. Baskaran. This is an open access article distributed under the terms of the Creative Commons License, which permits unrestricted use and distribution provided the original author and source are credited. 


\section{Introduction}

Let $\mathbf{f}(z)$ be a normalized analytic function of the form

$$
\mathbf{f}(z)=z+\sum_{n=2}^{\infty} a_{n} z^{n}, z \in \mathbf{U}, \mathbf{U}=\{z: z \in C,|z|<1\}
$$

and let $A$ be the class of all such functions. Let $S=\{f(z) \in A \ni$ $f(z)$ is univalent in $\mathbf{U}\}$.

For $\mathbf{f}_{\mathbf{1}}(z)$ and $\mathbf{f}_{\mathbf{2}}(z) \in A$, we say that $\mathbf{f}_{\mathbf{1}}(z)$ is subordinate to $\mathbf{f}_{\mathbf{2}}(z)$, if there exists a function namely, Schwarz function $w(z)$ with $w(0)=0$ and $|w(z)|<1$ in $\mathbf{U}$ such that $\mathbf{f}_{\mathbf{1}}(z)=\mathbf{f}_{\mathbf{2}}(w(z))$ and we write $\mathbf{f}_{\mathbf{1}}(z) \prec \mathbf{f}_{\mathbf{2}}(z)$.

Obtaining sharp bounds for $\left|a_{3}-\eta a_{2}^{2}\right|$ of any compact family of functions is called the Fekete-Szegö problem. In particular when $\eta=1$, the functional represents Schwarzian derivative and the role of Schwarzian derivative in the theory of Geometric functions is remarkable.

Let $\sigma$ denote the class of all bi-univalent functions in $\mathbf{U}$. We say that a function $\mathbf{f}(z)$ in $S$ belongs to $\sigma$, if both $\mathbf{f}(z)$ and its inverse has an analytic continuation to $|w|<1$.

Lewin [8] introduced the class of bi-univalent functions in 1967 and gave an estimate for the second coefficient for functions belonging to this class as $\left|a_{2}\right|<1.51$. His result was improved by Brannan and Clunie [3] to $\left|a_{2}\right| \leq \sqrt{2}$. There is an extensive literature on the estimates of the initial coefficients of bi-univalent functions (see $[10,12,13,14]$ ).

Horadam polynomials are generalized Horadam numbers and second order polynomial sequence. Recently, Horzum and Kocer [4], studied the Horadam polynomials $\mathbf{h}_{n}(x)$, which is given by the following recurrence relation [5]

$$
\mathbf{h}_{n}(x)=\varrho x \mathbf{h}_{n-1}(x)+\varrho \mathbf{h}_{n-2}(x), \mathbf{N}-\{1,2\} .
$$

One can refer to $[1,5,6,7,9]$, for more details. These polynomials and their generalizations play a vital role in Mathematics, Statistics and Physics. The first few Horadam polynomials are listed below:

$$
\mathbf{h}_{1}(x)=\mathbf{b}, \mathbf{h}_{2}(x)=\mathbf{a} x, \mathbf{h}_{3}(x)=\mathbf{a} \varrho x^{2}+\mathbf{b} p,
$$


for some real constants, $\mathbf{b}, \mathbf{a}, \varrho$ and $p$.

Following figure illustrates the special cases of Horadam polynomials

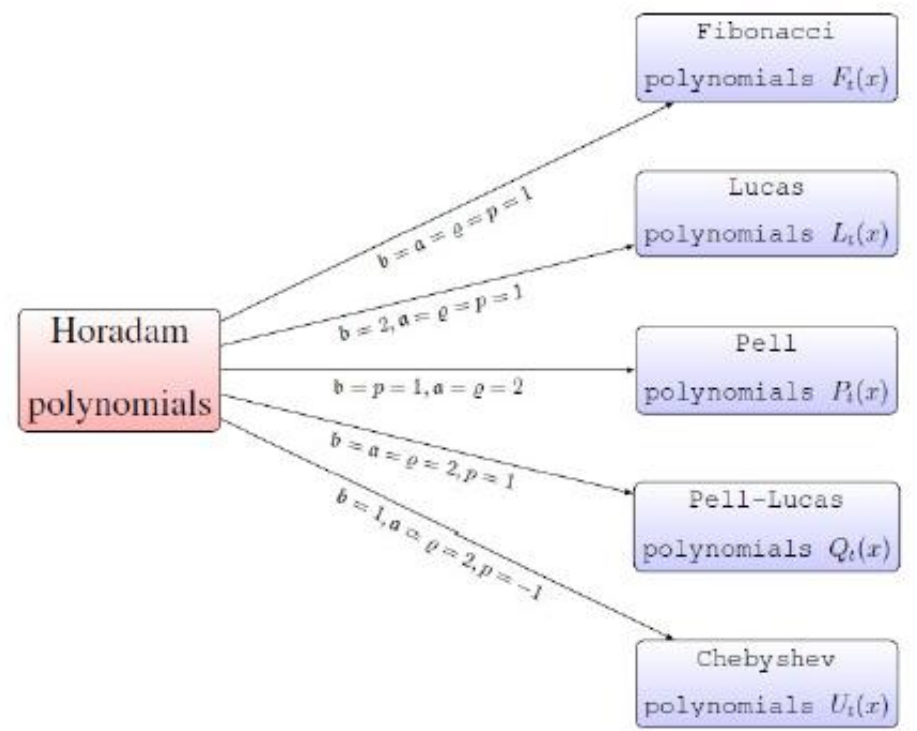

Figure 1:

Theorem 1.1. Let $\mathbf{G}(x, z)$ be the generating function of the Horadam polynomials $\mathbf{h}_{n}$. Then,

$$
\mathbf{G}(x, z)=\sum_{n=1}^{\infty} \mathbf{h}_{n}(x) z^{n-1}=\frac{\mathbf{b}+(\mathbf{a}-\mathbf{b} \varrho) x z}{1-\varrho x z-p z^{2}}
$$

Remark 1.1. [11] Choosing $\mathbf{b}=1, \mathbf{a}=\varrho=2, p=-1$ and $x \longrightarrow \eta$, in Theorem 1.1, the generating function $\mathbf{G}(x, z)$ generates the Chebyshev polynomials $U_{k}(\eta)$ of the second kind, which is given by

$$
U_{k}(\eta)=(k+1)_{2} F_{1}\left(-k, k+2 ; \frac{3}{2} ; \frac{1-\eta}{2}\right)=\frac{\sin (k+1) \varphi}{\sin \varphi},(\eta=\sin \varphi)
$$

in terms of the celebrated Gaussian hypergeometric function ${ }_{2} F_{1}$. 
Definition 1.1. A function $\mathbf{f} \in \sigma$ is said to be in the class

$$
\mathbf{A}_{\sigma}(\gamma, \delta ; x) \quad(\gamma \geq 0 ; 0 \leq \delta \leq 1 ; z, w \in \mathbf{U})
$$

if

$(1-\gamma)(1-\delta) \frac{\mathbf{f}(z)}{z}+(\delta+\gamma(1+\delta)) \mathbf{f}^{\prime}(z)+\gamma \delta\left(z \mathbf{f}^{\prime \prime}(z)-2\right) \prec 1-\mathbf{b}+\mathbf{G}(x, z)$

and

$(1-\gamma)(1-\delta) \frac{\mathbf{g}(w)}{w}+(\delta+\gamma(1+\delta)) \mathbf{g}^{\prime}(w)+\gamma \delta\left(w \mathbf{g}^{\prime \prime}(w)-2\right) \prec 1-\mathbf{b}+\mathbf{G}(x, w)$

where $\mathbf{g}=\mathbf{f}^{-1}$.

By choosing the parameters $\gamma$ and $\delta$ appropriately, the class reduces to known subclasses of bi-univalent functions.

Example 1.1. For $\delta=0$ and $\gamma \geq 1, \mathbf{f} \in \sigma$ is in the class

$$
\mathbf{A}_{\sigma}(\gamma, 0 ; x)=\mathbf{D}_{\sigma}(\gamma ; x) \quad(z, w \in \mathbf{U})
$$

if

$$
(1-\gamma) \frac{\mathbf{f}(z)}{z}+\gamma \mathbf{f}^{\prime}(z) \prec 1-\mathbf{b}+\mathbf{G}(x, z)
$$

and

$$
(1-\gamma) \frac{\mathbf{g}(w)}{w}+\gamma \mathbf{g}^{\prime}(w) \prec 1-\mathbf{b}+\mathbf{G}(x, w)
$$

where $\mathbf{g}=\mathbf{f}^{-1}$.

Example 1.2. For $\delta=0$ and $\gamma=1, \mathbf{f} \in \sigma$ is in the class

$$
\mathbf{A}_{\sigma}(1,0 ; x)=\mathbf{D}_{\sigma}(1 ; x)=\mathbf{H}_{\sigma}(x) \quad(z, w \in \mathbf{U})
$$

if

$$
\mathbf{f}^{\prime}(z) \prec 1-\mathbf{b}+\mathbf{G}(x, z)
$$

and

$$
\mathbf{g}^{\prime}(w) \prec 1-\mathbf{b}+\mathbf{G}(x, w)
$$

where $\mathbf{g}=\mathbf{f}^{-1}$. The class $\mathbf{H}_{\sigma}(x)$ was investigated and studied by Alamous $[2]$ 


\section{Coefficient Bounds}

Theorem 2.1. Let $\mathbf{f}$ represented by 1.1 belong to the class $\mathbf{A}_{\sigma}(\gamma, t ; x)$. Then,

$$
\left|a_{2}\right| \leq \frac{|\mathbf{a} x| \sqrt{|\mathbf{a} x|}}{\sqrt{\left|(1+2(\gamma+\delta)+10 \gamma \delta)(\mathbf{a} x)^{2}-(1+(\gamma+\delta)+5 \gamma \delta)^{2}\left(\mathbf{a} \rho x^{2}+\mathbf{b} p\right)\right|}}
$$

and

$$
\left|a_{3}\right| \leq \frac{|\mathbf{a} x|}{(1+2(\gamma+\delta)+10 \gamma \delta)}+\frac{\mathbf{a}^{2} x^{2}}{(1+(\gamma+\delta)+5 \gamma \delta)^{2}}
$$

Proof. Consider $\mathbf{f} \in \mathbf{A}_{\sigma}(\gamma, \delta ; x)$. According to Definition 1.1, there exists two analytic functions $\Theta$ and $\Upsilon$ with $\Theta(0)=\Upsilon(0)=0,|\Theta(z)|<$ $1,|\Upsilon(w)|<1$ for all $z, w \in \mathbf{U}$ such that

$(1-\gamma)(1-\delta) \frac{\mathbf{f}(z)}{z}+(\delta+\gamma(1+\delta)) \mathbf{f}^{\prime}(z)+\gamma \delta\left(z \mathbf{f}^{\prime \prime}(z)-2\right) \prec 1-\mathbf{b}+\mathbf{G}(x, \Theta(z))$

and

$(1-\gamma)(1-\delta) \frac{\mathbf{g}(w)}{w}+(\delta+\gamma(1+\delta)) \mathbf{g}^{\prime}(w)+\gamma \delta\left(w \mathbf{g}^{\prime \prime}(w)-2\right) \prec 1-\mathbf{b}+\mathbf{G}(x, \Upsilon(w))$,

or equivalently

$$
\begin{gathered}
(1-\gamma)(1-\delta) \frac{\mathbf{f}(z)}{z}+(\delta+\gamma(1+\delta)) \mathbf{f}^{\prime}(z)+\gamma \delta\left(z \mathbf{f}^{\prime \prime}(z)-2\right) \\
=1+\mathbf{h}_{1}(x)-\mathbf{b}+\mathbf{h}_{2}(x) \Theta(z)+\mathbf{h}_{3} \Theta^{2}(z)+\cdots \\
(1-\gamma)(1-\delta) \frac{\mathbf{g}(w)}{w}+(\delta+\gamma(1+\delta)) \mathbf{g}^{\prime}(w)+\gamma \delta\left(w \mathbf{g}^{\prime \prime}(w)-2\right) \\
=1+\mathbf{h}_{1}(x)-\mathbf{b}+\mathbf{h}_{2}(x) \Upsilon(w)+\mathbf{h}_{3} \Upsilon^{2}(w)+\cdots
\end{gathered}
$$

2.1 and 2.2 yield

$$
\begin{gathered}
(1-\gamma)(1-\delta) \frac{\mathbf{f}(z)}{z}+(\delta+\gamma(1+\delta)) \mathbf{f}^{\prime}(z)+\gamma \delta\left(z \mathbf{f}^{\prime \prime}(z)-2\right) \\
=1+\mathbf{h}_{2}(x) u_{1} z+\left[\mathbf{h}_{2}(x) u_{2}+\mathbf{h}_{3}(x) u_{1}^{2}\right] z^{2}+\cdots \\
(1-\gamma)(1-\delta) \frac{\mathbf{g}(w)}{w}+(\delta+\gamma(1+\delta)) \mathbf{g}^{\prime}(w)+\gamma \delta\left(w \mathbf{g}^{\prime \prime}(w)-2\right) \\
=1+\mathbf{h}_{2}(x) v_{1} w+\left[\mathbf{h}_{2}(x) v_{2}+\mathbf{h}_{3}(x) v_{1}^{2}\right] w^{2}+\cdots
\end{gathered}
$$

It is to be noted that if

$$
|\Theta(z)|=\left|u_{1} z+u_{2} z^{2}+u_{3} z^{3}+\cdots\right|<1 \quad(z \in \mathbf{U})
$$


and

$$
|\Upsilon(w)|=\left|v_{1} w+v_{2} w^{2}+v_{3} w^{3}+\cdots\right|<1 \quad(w \in \mathbf{U})
$$

then

$$
\begin{aligned}
\left|u_{k}\right| & \leq 1 \\
\left|v_{k}\right| & \leq 1 \quad(k \in \mathbf{N}) .
\end{aligned}
$$

Comparing the corresponding coefficients in 2.3 and 2.4, we have

$$
\begin{gathered}
(1+(\gamma+\delta)+5 \gamma \delta) a_{2}=\mathbf{h}_{2}(x) u_{1} \\
(1+2(\gamma+\delta)+10 \gamma \delta) a_{3}=\mathbf{h}_{2}(x) \mathbf{u}_{2}+\mathbf{h}_{3}(x) u_{1}^{2} \\
-(1+(\gamma+\delta)+5 \gamma \delta) a_{2}=\mathbf{h}_{2}(x) v_{1}
\end{gathered}
$$

and

$$
(1+2(\gamma+\delta)+10 \gamma \delta)\left(2 a_{2}^{2}-a_{3}\right)=\mathbf{h}_{2}(x) v_{2}+\mathbf{h}_{3}(x) v_{1}^{2}
$$

From Eqs. 2.5 and 2.7, we can easily see that

$$
\begin{gathered}
u_{1}=-v_{1} \\
2(1+(\gamma+\delta)+5 \gamma \delta)^{2} a_{2}^{2}=\mathbf{h}_{2}^{2}\left[u_{1}^{2}+v_{1}^{2}\right]
\end{gathered}
$$

If we add 2.6 to 2.8 , we get

$(2.11) 2(1+2(\gamma+\delta)+10 \gamma \delta) a_{2}^{2}=\mathbf{h}_{2}(x)\left(u_{2}+v_{2}\right)+\mathbf{h}_{3}(x)\left(u_{1}^{2}+v_{1}^{2}\right)$

By using 2.10 in the equality 2.11, we have

$2\left((1+2(\gamma+\delta)+10 \gamma \delta) \mathbf{h}_{2}^{2}(x)-(1+(\gamma+\delta)+5 \gamma \delta)^{2} \mathbf{h}_{3}(x)\right) a_{2}^{2}=\mathbf{h}_{2}^{3}\left(u_{2}+v_{2}\right)$ (2.12)

which implies

$$
\left|a_{2}\right| \leq \frac{|\mathbf{a} x| \sqrt{|\mathbf{a} x|}}{\sqrt{\left|(1+2(\gamma+\delta)+10 \gamma \delta)(\mathbf{a} x)^{2}-(1+(\gamma+\delta)+5 \gamma \delta)^{2}\left(\mathbf{a} \rho x^{2}+\mathbf{b} p\right)\right|}}
$$


Moreover, if we subtract 2.8 from 2.6 and use 2.9,

$$
a_{3}-a_{2}^{2}=\frac{\mathbf{h}_{2}(x)\left(u_{2}-v_{2}\right)}{2(1+2(\gamma+\delta)+10 \gamma \delta)}
$$

Then, in view of 2.10 and 2.13 , we have

$$
a_{3}=\frac{\mathbf{h}_{2}(x)\left(u_{2}-v_{2}\right)}{2(1+2(\gamma+\delta)+10 \gamma \delta)}+\frac{\mathbf{h}_{2}^{2}(x)\left(u_{1}^{2}+v_{1}^{2}\right)}{2(1+(\gamma+\delta)+5 \gamma \delta)^{2}}
$$

Applying $\mathbf{h}_{2}(x)$ and taking modulus, we deduce that

$$
\left|a_{3}\right| \leq \frac{|\mathbf{a} x|}{(1+2(\gamma+\delta)+10 \gamma \delta)}+\frac{\mathbf{a}^{2} x^{2}}{(1+(\gamma+\delta)+5 \gamma \delta)^{2}}
$$

Corollary 2.1. Let $\mathbf{f} \in \mathbf{D}_{\sigma}(\gamma ; x)$. Then

$$
\begin{gathered}
\left|a_{2}\right| \leq \frac{|\mathbf{a} x| \sqrt{|\mathbf{a} x|}}{\sqrt{\left|(1+2 \gamma)(\mathbf{a} x)^{2}-(1+\gamma)^{2}\left(\mathbf{a} \rho x^{2}+\mathbf{b} p\right)\right|}} \\
\left|a_{3}\right| \leq \frac{|\mathbf{a} x|}{(1+2 \gamma)}+\frac{\mathbf{a}^{2} x^{2}}{(1+\gamma)^{2}}
\end{gathered}
$$

Corollary 2.2. Let $\mathbf{f} \in \mathbf{H}_{\sigma}(x)$. Then

$$
\begin{gathered}
\left|a_{2}\right| \leq \frac{|\mathbf{a} x| \sqrt{|\mathbf{a} x|}}{\sqrt{\left|3(\mathbf{a} x)^{2}-4\left(\mathbf{a} \rho x^{2}+\mathbf{b} p\right)\right|}} \\
\left|a_{3}\right| \leq \frac{|\mathbf{a} x|}{3}+\frac{\mathbf{a}^{2} x^{2}}{4}
\end{gathered}
$$

The class was investigated and studied by Alamous [2].

Corollary 2.3. For $\eta \in\left(\frac{1}{2}, 1\right)$, let the function $\mathbf{f} \in \mathbf{A}_{\sigma}(\gamma, \delta ; \eta)$ be of the form 1.1. Then

$$
\begin{gathered}
\left|a_{2}\right| \leq \frac{|2 \eta| \sqrt{|2 \eta|}}{\sqrt{\left|4 \eta^{2}(1+2(\gamma+\delta)+10 \gamma \delta)-\left(4 \eta^{2}-1\right)(1+(\gamma+\delta)+5 \gamma \delta)^{2}\right|}} \\
\left|a_{3}\right| \leq \frac{|2 \eta|}{(1+2(\gamma+\delta)+10 \gamma \delta)}+\frac{4 \eta^{2}}{(1+(\gamma+\delta)+5 \gamma \delta)^{2}}
\end{gathered}
$$




\section{Fekete-Szegö Inequality}

In this section, for functions belonging to the class $\mathbf{A}_{\sigma}(\gamma, t ; x)$, we have estimated the bounds for the linear functional.

Theorem 3.1. Let $\mathbf{f} \in \mathbf{A}_{\sigma}(\gamma, t ; x)$ and $\zeta \in \mathbf{R}$. Then,

$$
\left|a_{3}-\zeta a_{2}^{2}\right| \leq\left\{\begin{array}{cl}
\frac{|\mathbf{a} x|}{s_{1}}, & |\zeta-1| \leq \mid 1-\frac{s_{2}^{2}\left(\mathbf{a} \rho x^{2}+\mathbf{b} p\right)}{s_{1} \mathbf{a}^{2} x^{2}} \\
\frac{|\zeta-1||\mathbf{a} x|^{3}}{\left.\mid s_{1} a-s_{2}^{2} \rho\right] \mathbf{a} x^{2}-s_{2}^{2} \mathbf{b} p}, & |\zeta-1| \geq\left|1-\frac{s_{2}^{2}\left(\mathbf{a} \rho x^{2}+\mathbf{b} p\right)}{s_{1} \mathbf{a}^{2} x^{2}}\right|
\end{array} \mid\right.
$$

where,

$$
\begin{gathered}
s_{1}=1+2(\gamma+\delta)+10 \gamma \delta \\
s_{2}=1+(\gamma+\delta)+5 \gamma \delta
\end{gathered}
$$

Proof. From 2.13, for $\zeta \in \mathbf{R}$, we have

$$
a_{3}-\zeta a_{2}^{2}=\frac{\mathbf{h}_{2}(x)\left(u_{2}-v_{2}\right)}{2(1+2(\gamma+\delta)+10 \gamma \delta)}+(1-\zeta) a_{2}^{2}
$$

By using 2.12 in 3.1, we have

$$
\begin{aligned}
& a_{3}-\zeta a_{2}^{2}=\frac{\mathbf{h}_{2}(x)\left(u_{2}-v_{2}\right)}{2(1+2(\gamma+\delta)+10 \gamma \delta)}+(1-\zeta)\left(\frac{\mathbf{h}_{2}^{3}(x)\left(u_{2}+v_{2}\right)}{2\left[(1+2(\gamma+\delta)+10 \gamma \delta] \mathbf{h}_{2}^{2}(x)-(1+\gamma+\delta+5 \gamma \delta)^{2} \mathbf{h}_{3}(x)\right.}\right) \\
& =\mathbf{h}_{2}(x)\left[\left(\frac{1}{2[1+2(\gamma+\delta)+10 \gamma \delta]}+\chi(\gamma, \delta)\right) u_{2}+\left(\frac{-1}{2[1+2(\gamma+\delta)+10 \gamma \delta]}+\chi(\gamma, \delta)\right) v_{2}\right]
\end{aligned}
$$

where $\chi(\gamma, \delta)=\frac{(1-\zeta) \mathbf{h}_{2}^{2}(x)}{2\left[(1+2(\gamma+\delta)+10 \gamma \delta) \mathbf{h}_{2}^{2}(x)-(1+(\gamma+\delta)+5 \gamma \delta)^{2} \mathbf{h}_{3}(x)\right.}$

Then, in view of (1.2), we conclude that

$$
\left|a_{3}-\zeta a_{2}^{2}\right| \leq \begin{cases}\frac{\left|h_{2}(x)\right|}{1+2(\gamma+\delta)+10 \gamma \delta}, & 0 \leq|\chi(\gamma, \delta)| \leq \frac{1}{2[1+2(\gamma+\delta)+10 \gamma \delta]} \\ 2\left|\mathbf{h}_{2}(x)\right||\chi(\gamma, \delta)|, & |\chi(\gamma, \delta)| \geq \frac{1}{2[1+2(\gamma+\delta)+10 \gamma \delta]}\end{cases}
$$


Corollary 3.1. Let $\mathbf{f} \in \mathbf{D}_{\sigma}(\gamma ; x)$ and $\zeta \in \mathbf{R}$. Then

$$
\left|a_{3}-\zeta a_{2}^{2}\right| \leq \begin{cases}\frac{|\mathbf{a} x|}{1+2 \gamma}, & |\zeta-1| \leq\left|1-\frac{(1+\gamma)^{2}\left(\mathbf{a} \varrho x^{2}+\mathbf{b} p\right)}{(1+2 \gamma) \mathbf{a}^{2} x^{2}}\right| . \\ \frac{|1-\zeta \| \mathbf{a} x|^{3}}{\left.\pi(1+2 \gamma) \mathbf{a}-(1+\gamma)^{2} \varrho\right] \mathbf{a} x^{2}-[1+\gamma]^{2} \mathbf{b} p \mid}, & |\zeta-1| \geq\left|1-\frac{(1+\gamma)^{2}\left(\mathbf{a} \varrho x^{2}+\mathbf{b} p\right)}{(1+2 \gamma) \mathbf{a}^{2} x^{2}}\right| .\end{cases}
$$

Corollary 3.2. Let $\mathbf{f} \in \mathbf{H}_{\sigma}(\gamma ; x)$ and $\zeta \in \mathbf{R}$. Then

$$
\left|a_{3}-\zeta a_{2}^{2}\right| \leq \begin{cases}\frac{|\mathbf{a} x|}{3}, & |\zeta-1| \leq\left|1-\frac{4\left(\mathbf{a} \varrho x^{2}\right)}{3 \mathbf{a}^{2} x^{2}}\right| \\ \frac{\left|\mathbf{a} x x^{3}\right| \zeta-1 \mid}{\left|[3 \mathbf{a}-4 \varrho] \mathbf{a} x^{2}-4 \mathbf{b} p\right|,} & |\zeta-1| \geq\left|1-\frac{4\left(\mathbf{a} \varrho x^{2}\right)}{3 \mathbf{a}^{2} x^{2}}\right|\end{cases}
$$

Corollary 3.3. For $\eta \in\left(\frac{1}{2}, 1\right)$, let the function $\mathbf{A}_{\sigma}(\gamma, \delta ; \eta)$ be of the form 1.1. Then

$$
\left|a_{3}-\zeta a_{2}^{2}\right| \leq\left\{\begin{array}{cl}
\frac{|2 \eta|}{s_{1}}, & |\zeta-1| \leq\left|1 \frac{s_{2}^{2}\left(4 \eta^{2}-0\right)}{4 s_{1} \eta^{2}}\right| \\
\frac{|\zeta-1||2 \eta|^{3}}{\left|4\left[s_{1}-s_{2}^{2}\right] \eta^{2}+s_{2}^{2}\right|,} & |\zeta-1| \geq\left|1-\frac{s_{2}^{2}\left(4 \eta^{2}-1\right)}{4 s_{1} \eta^{2}}\right|
\end{array}\right.
$$

where,

$$
\begin{gathered}
s_{1}=1+2(\gamma+\delta)+10 \gamma \delta \\
s_{2}=1+(\gamma+\delta)+5 \gamma \delta
\end{gathered}
$$

\section{References}

[1] A. Akgül and F. Sakar, "A certain subclass of bi-univalent analytic functions introduced by means of the q-analogue of Noor integral operator and Horadam polynomials", Turkish journal of mathematics, vol. 43, no. 5, pp. 2275-2286, 2019, doi: 10.3906/ mat-1905-17

[2] A. G. Alamoush, "Coefficient estimates for certain subclass of bi functions associated the Horadam polynomials", 2018, arXiv: 1812.10589 
[3] D. Brannan and J. Clunie, Aspects of contemporary complex analysis. London: Academic Press, 1980.

[4] T. Horzum and E. Gökçen Koçer, "On some properties of Horadam polynomials", International mathematical forum, vol. 4, no. 25, pp. 1243-1252, 2009. [On line]. Available: https:/ / bit.ly/ 2Sw3MGf

[5] A. F. Horadam and J. M. Mahon, "Pell and Pell-Lucas polynomials", The Fibonacci quarterly, vol. 23, no. 1, pp. 7-20, 1985. [On line]. Available: https:/ / bit.ly/ 2R137MV

[6] A. Horadam, "Jacobsthal representation polynomials", The Fibonacci quarterly, vol. 35, no. 2, pp. 137-148, 1997. [On line]. Available: https:/ / bit.ly/ 3ez7OWY

[7] T. Koshy, Fibonacci and Lucas numbers with applications. New York, NY: Wiley, 2001, doi: 10.1002/ 9781118033067

[8] M. Lewin, "On a coefficient problem for bi-univalent functions", Proceedings of the American Mathematical Society, vol. 18, no. 1, pp. 63-63, 1967, doi: 10.1090/ s0002-9939-1967-0206255-1

[9] A. Lupas, "A Guide of Fibonacci and Lucas polynomials", Octogon Mathematical Magazine, vol. 7, no. 1, pp. 2-12, 1999.

[10] G. Saravanan and K. Muthunagai, "Estimation of upper bounds for initial coefficients and Fekete-Szegö inequality for a subclass of analytic bi-univalent functions", in Applied mathematics and scientific computing, B. Rushi Kumar, R. Sivaraj, B. S. R. V. Prasad, M. Nalliah, and A. Subramanyam Reddy, Eds. Basel: Birkhäuser, 2019, pp. 57-65, doi: 10.1007/978-3-030-01123-9 7

[11] G. Szegö, Orthogonal polynomials, 4th ed. Providence, RI: AMS, 1975.

[12] R. Vijaya, T. Sudharsan and S. Sivasubramanian, "Coefficient estimates for certain subclasses of biunivalent functions defined by convolution", International journal of analysis, vol. 2016, Art. ID. 6958098, 2016, doi: $10.1155 / 2016 / 6958098$

[13] Q. Xu, Y. Gui, and H. Srivastava, "Coefficient estimates for a certain subclass of analytic and bi-univalent functions", Applied mathematics letters, vol. 25, no. 6, pp. 990-994, 2012, doi: 10.1016/j.aml.2011. 11.013

[14] S. Yalçin, K. Muthunagai and G. Saravanan, "A subclass with bi-univalence involving $(p, q)$ - Lucas polynomials and its coefficient bounds", Boletín de la Sociedad Matemática Mexicana, vol. 26, pp. 1015-1022, doi: 10.1007/s40590-020-00294-z 